\title{
Utilization of Kidneys With Similar Kidney Donor Risk Index Values From Standard Versus Expanded Criteria Donors
}

\author{
K. J. Woodside ${ }^{a, *}$, R. M. Merion ${ }^{\mathrm{b}, \mathrm{c}}$, A. B. \\ Leichtman ${ }^{\mathrm{c}, \mathrm{d}}$, R. de los Santos ${ }^{\mathrm{c}}$, C. J. Arrington ${ }^{\mathrm{c}}$, \\ P. S. Rao ${ }^{c, d}$ and R. S. Sung ${ }^{b, c}$ \\ a Department of Surgery, Case Western Reserve \\ University, Cleveland, $\mathrm{OH}$ \\ ${ }^{\mathrm{b}}$ Department of Surgery, University of Michigan, Ann \\ Arbor, MI \\ ${ }^{\mathrm{C}}$ Arbor Research Collaborative for Health, Ann Arbor, MI \\ dDepartment of Internal Medicine, University of Michigan, \\ Ann Arbor, MI \\ * Correspondence: Kenneth J. Woodside, \\ kenneth.woodside@uhhospitals.org
}

With the shortage of standard criteria donor (SCD) kidneys, efficient expanded criteria donor (ECD) kidney utilization has become more vital. We investigated the effects of the ECD label on kidney recovery, utilization and outcomes. Using data from the Scientific Registry of Transplant Recipients from November 2002 to May 2010, we determined recovery and transplant rates, and modeled discard risk, for kidneys within a range of kidney donor risk index (KDRI) 1.4-2.1 that included both SCD and ECD kidneys. To further compare similar quality kidneys, these kidneys were again divided into three KDRI intervals. Overall, ECD kidneys had higher recovery rates, but lower transplant rates. However, within each KDRI interval, SCD and ECD kidneys were transplanted at similar rates. Overall, there was increased risk for discard for biopsied kidneys. SCD kidneys in the lower two KDRI intervals had the highest risk of discard if biopsied. Pumped kidneys had a lower risk of discard, which was modulated by KDRI for SCD kidneys but not ECD kidneys. Although overall ECD graft survival was worse than SCD, there were no differences within individual KDRI intervals. Thus, ECD designation adversely affects neither utilization nor outcomes beyond that predicted by KDRI.

Key words: Expanded criteria donor (ECD), kidney donor risk index (KDRI), kidney transplantation, outcomes, registry, utilization

Abbreviations: CMV, cytomegalovirus; ECD, expanded criteria donor; HBcAb, hepatitis B core antibody; HBsAg, hepatitis B surface antigen; HCV, hepatitis C virus; HLA, human leukocyte antigen; HTLV, human T-lymphotrophic virus; HRSA, Health Resources and Services Administration; KDRI, kidney donor risk index; PRA, panel reactive antibody; OPO, organ procurement organization; OR, odds ratio; SCD, standard criteria donor; SRTR, Scientific Registry of Transplant Recipients.

Received 31 January 2012, revised 16 March 2012 and accepted for publication 10 April 2012

\section{Introduction}

With the ongoing organ shortage, efforts to expand the organ pool have intensified. This has included use of expanded criteria donors (ECDs), defined as all donors age 60 years or older, or those age $50-59$ years who meet two or more of the following criteria: serum creatinine $>1.5$ $\mathrm{mg} / \mathrm{dL}$, cerebrovascular accident as the cause of death or history of hypertension (1). While ECD kidneys, as a group, have inferior outcomes when compared to kidneys from donors that do not meet the ECD definition (2), many of these kidneys are potentially utilizable organs with acceptable outcomes. As the ECD designation suggests an increased risk of inferior outcomes, many centers do not list patients for ECD kidneys (3), despite a probable benefit from ECD listing for a significant proportion of these patients (4). This "labeling effect" has been hypothesized in part based on analyses that suggest that ECD kidneys are recovered more frequently than before the adoption of the ECD allocation system, but are less likely to be utilized (5).

As there is a continuum of quality for donated kidneys, and the standard criteria donors (SCD)/ECD designation is binary, a number of postprocurement processes have been implemented in efforts to increase ECD utilization while preserving outcomes, such as pulsatile perfusion and kidney biopsy. Preprocurement risk stratification is more useful in that it is less costly and more efficient. To this end, Rao et al. have developed a kidney donor risk index (KDRI) that evaluates all donor kidneys along a continuum of risk (6). The KDRI incorporates, in addition to the ECD risk factors, a number of other parameters that impact graft survival, including donor factors such as diabetes, height, weight, donation after cardiac death (DCD) status, hepatitis C status, and transplant factors such as HLA mismatch and en bloc or double kidney transplant. Within this formula, 
as the KDRI score increases, the percentage of ECDs that make up a given score range increases, as these tend to be higher risk donor kidneys. However, considerable overlap exists between the distributions of SCD and ECD kidneys on the KDRI spectrum, further highlighting the limitations of the ECD designation in defining donor quality.

To address whether an ECD "labeling effect" exists, we studied whether the ECD designation was associated with increased risk of discard and adverse transplant outcomes when compared to SCD kidneys of similar quality based on the KDRI. While utilization and acceptance behavior with respect to ECD labeling has been studied, it is not known if kidneys with similar risk, but a different label (i.e. high KDRI kidneys in the SCD spectrum vs. similar KDRI kidneys with the ECD label) have different acceptance patterns.

\section{Methods}

\section{Sample}

We used data from the Scientific Registry of Transplant Recipients (SRTR) on kidneys from deceased donors 18 years or older from November 1 , 2002 to May 31, 2010 with extra ascertainment of graft failure and death from the Centers for Medicare \& Medicaid Services and the Social Security Death Master File, respectively. From this sample, we then identified the organs that were recovered and transplanted, and obtained for each the corresponding recipient information. Recipient data were left missing for organs that were recovered but not transplanted (i.e. discarded).

We arrived at our final sample in a stepwise fashion. First, because the objectives of this study required the use of the KDRI, we aimed to make our data similar to the data used in the initial formulation of the KDRI. We applied certain exclusions to the data to achieve this goal. Among transplanted organs, the exceptions were recipients less than 18 years old, previous transplant, multiorgan transplant, $A B O$ incompatible with the donor, or donors with missing height, weight or creatinine level. Next, since we were only interested in comparing two donor classifications, we further limited our sample to include only organs from ECD and SCD (i.e. DCD donors were excluded).

We calculated the KDRI, categorized it into deciles, and plotted it by donor type (ECD vs. SCD). Afterward, we identified a region within which a substantial number of kidneys have the same risk index but were classified differently (i.e. two kidneys with the same value for KDRI but one was labeled ECD, whereas the other was labeled SCD). There were 23203 kidneys that fell within the selected region, of which 20246 (87.3\%) were actually recovered for transplant. Of the recovered kidneys, 13833 (68.3\%) were transplanted, whereas $6413(31.7 \%)$ were discarded. This KDRI overlap region was further divided into three interval categories $(\geq 1.4$ to $\leq 1.6$, $>1.6$ to $\leq 1.8$ and $>1.8$ to $\leq 2.1$, which represented $37.6,30.5$ and $31.9 \%$ of the recovered kidneys, respectively).

\section{Variables}

Donor type: ECD kidneys are defined as kidneys from deceased donors aged 50-59 years with at least two of three of the following conditions: (1) history of hypertension, (2) serum creatinine $>1.5 \mathrm{mg} / \mathrm{dL}$ and (3) death caused by cerebrovascular accident; or donors aged 60 or older regardless of the presence of comorbid conditions (1). For this analysis, we excluded kidneys from all DCD donors, as well as en bloc and double kidney transplants.
Kidney donor risk index: The KDRI is derived from a Cox regression model adjusting for various donor and transplant factors that were identified to be significant in a stepwise variable deletion process (6). KDRI is computed using the estimates of each factor that applies to a particular deceased donor kidney (6), including the following: age, race, history of diabetes or hypertension, serum creatinine, height, weight, hepatitis $\mathrm{C}$ virus $(\mathrm{HCV})$ result and cause of death. Because some of our analyses include all recovered kidneys, whether or not they were transplanted, and because we were focusing on the effect of donor quality, we set the KDRI equation components for DCD and en bloc/double kidney (which were excluded) and for the other transplant-related variables (e.g. HLA mismatch and cold ischemia time) to the value for the reference donor $(K D R I=1)$ so that these components did not contribute to variations in the KDRI.

\section{Analyses}

Discard model: We modeled the probability of discard of ECD and SCD kidneys within each of the previously defined KDRI intervals using logistic regression. The models were also adjusted for factors not in the KDRI or ECD definitions that could have potential effects on organ discard. We adjusted for donor sex (48.3\% males), blood type (48.7\% type O, 36\% type A, $11.8 \%$ type $B$ and $3.5 \%$ type $A B$ ) and lifestyle habits such as having a tattoo $(11.1 \%)$, alcohol consumption $(22.1 \%)$ and use of cocaine $(10.9 \%)$ or other drugs (17.9\%). The majority of the data on cigarette use are missing; hence, we adjusted instead for missing information on cigarette use $(54.3 \%)$. Serology results were also factored in the analysis. For instance, positivity on cytomegalovirus ( $\mathrm{CMV}, 70.8 \%)$, hepatitis B core antibody (HBcAb, 9.2\%) and human T cell lymphotrophic virus (HTLV, $0.1 \%$ ) were utilized as covariates. Furthermore, we adjusted for whether kidneys were pumped $(30.7 \%)$ or biopsied $(78.9 \%)$.

Donors or recipient records with missing data (3.8\% of total sample) were dropped from the model (unless categorized as missing). Models were stratified by donor type $(E C D=59.2 \%, S C D=40.9 \%)$ and $K D R I$ category. We also examined supplementary models that tested for trends-these models included all kidneys in the cohort (KDRI 1.4-2.1) but included an interaction term with the KDRI interval category.

Patient survival and graft failure models: We fitted a Cox regression to estimate the relative rate of patient death and graft failure among recipients. Patient follow-up began on the day of transplant up to 5 years posttransplant. Graft failure is defined as the earliest of retransplantation, return to dialysis or patient death. In total, there were $1985(14.4 \%)$ patient deaths and 3255 $(23.5 \%)$ graft failures out of 13833 transplanted organs. Both models were adjusted for recipient characteristics such as sex, age, race and diabetes. Initial steps were undertaken to assess whether the proportional hazards assumption was met for each of the covariates. Means were assigned as baseline values.

Similar to the discard model, the KDRI used in both the patient sur vival and graft failure models was the categorized KDRI (1.4-1.6, 1.6-1.8, 1.8-2.1). A model was fitted for each KDRI category, stratified by donor type. Stratifying our analysis allowed the survival rates to vary between strata, but assumed that the regression coefficients were the same across strata.

All analyses were performed using SAS version 9.1.3 (SAS Institute; Cary, NC, USA). Health Resources and Services Administration (HRSA) has determined that this study satisfies the criteria for the institutional review board exemption described in the "Public Benefit and Service Program" provisions of 45 Code of Federal Regulations 46.101 (b) (5) and HRSA Circular 03 


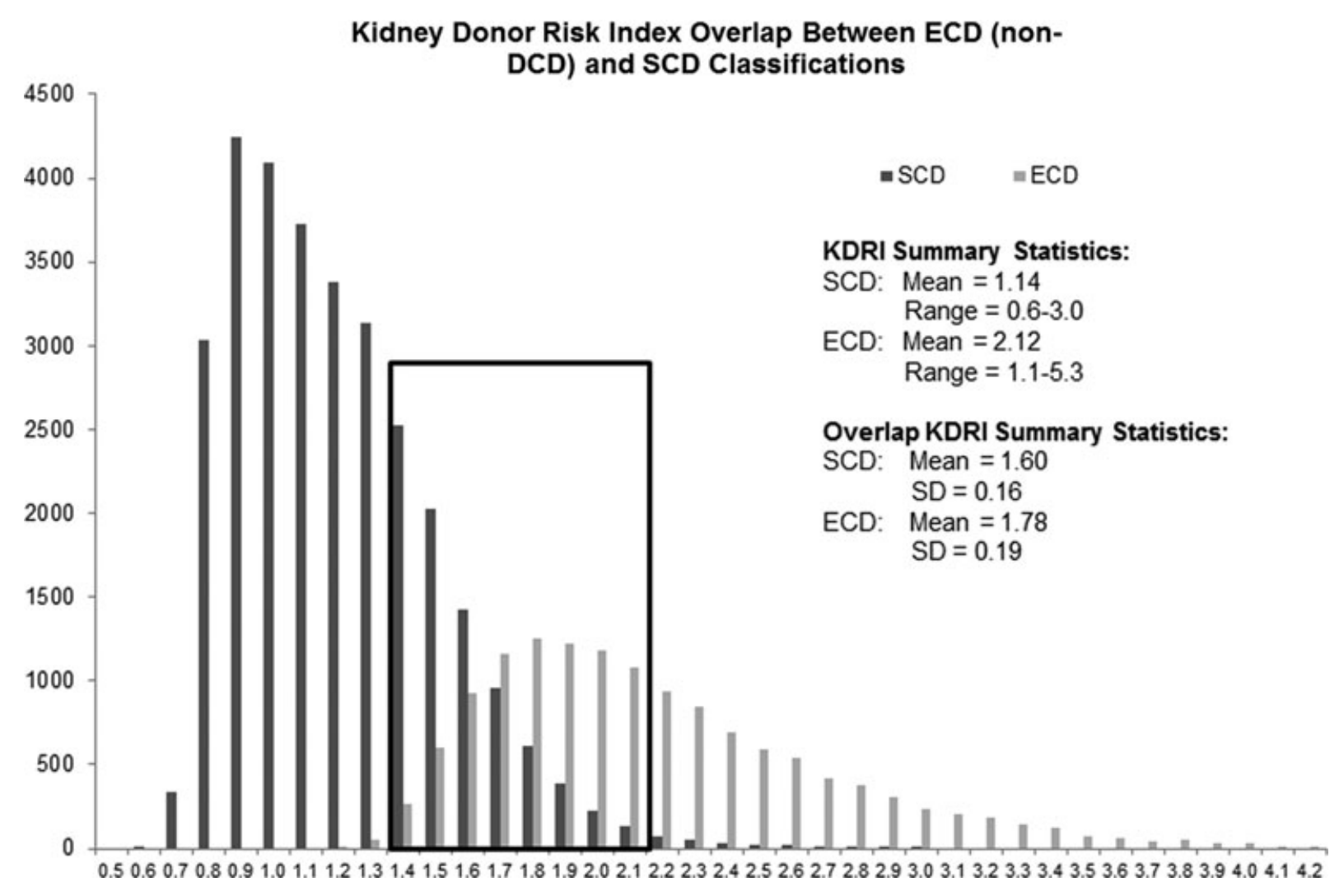

Figure 1: Distribution of ECD and SCD kidney donors. The boxed area represents the overlap region.

\section{Results}

This study focused on the subset of kidneys coming from a range of KDRI values that encompassed a significant number of both SCD and ECD kidneys (Figure 1). This "overlap region" was composed of 23203 kidneys, of which 20246 kidneys were recovered $(59 \%$ were ECD and $41 \%$ SCD). Within this cohort, ECD kidneys tended to have higher KDRI and SCD kidneys tended to have lower $K D R I$. The ECD kidneys in the overlap region were the lower risk ECD kidneys, whereas the overlap SCD kidneys were the upper range of risk for that group. Thus, this KDRI overlap region was further divided into three categories $(\geq 1.4$ to $\leq 1.6,>1.6$ to $\leq 1.8$ and $>1.8$ to $\leq 2.1)$ in efforts to more precisely compare SCD and ECD kidneys of similar quality.

Recipient demographics for the cohort by SCD and ECD classification are shown in Table 1. Patients receiving ECD kidneys were older and more likely to be white. There was a small, but statistically significant, trend for more male recipients in the ECD group. There were fewer ECD patients with glomerular disease, and more with diabetes, as the listed primary etiology of renal failure. Those receiving SCD kidneys had higher panel reactive antibody (PRA) values, both historically and at transplant, and fewer HLA mismatches, both collectively and at each measured HLA locus. There was a higher percentage of ECD recipients in the A blood group, and lower in the B blood group. Cold ischemia time was similar between the two groups.

Within the overlap range, a higher percentage of ECD kidneys than SCD kidneys were recovered $(89.7 \%$ vs. $84.0 \%$, $\mathrm{p}<0.0001$ ); this trend was maintained within each KDRI category (Table 2), suggesting a willingness by organ procurement organizations (OPOs) and transplant centers to recover ECD kidneys. The proportion of kidneys transplanted decreased with increasing KDRI category, and was lower for ECD kidneys overall $(66.1 \%$ vs. $71.6 \%$ for SCD kidneys, $p<0.0001)$. However, the proportion transplanted, defined as organs transplanted among those recovered, was similar for ECD and SCD kidneys in the lower and higher KDRI categories, and, paradoxically, was significantly greater for ECD kidneys in the 1.6-1.8 category $(71.5 \%$ vs. $63.4 \%$ for SCD kidneys, $p<0.0001)$, suggesting that the ECD designation does not appear to adversely affect utilization beyond that predicted by KDRI.

Adjusted models for discard were created for all kidneys in the overlap range, and were also stratified by KDRI interval (1.4-1.6, 1.6-1.8, 1.8-2.1) (Table 3). ECD kidneys were more likely to be discarded than SCD kidneys over the entire overlap region (odds ratio $[\mathrm{OR}]=1.29, \mathrm{p}<0.0001$ ). However, within each KDRI interval, ECD kidneys were not 
KDRI Modulates SCD \& ECD Utilization

Table 1: Recipient demographics

\begin{tabular}{|c|c|c|c|}
\hline & SCD & ECD & $\mathrm{p}$ \\
\hline Age (Mean \pm SD in years) & $52.7 \pm 11.9$ & $59.1 \pm 10.5$ & $<0.0001$ \\
\hline Sex & & & 0.04 \\
\hline Male & $3669(62.0 \%)$ & $5043(63.7 \%)$ & \\
\hline Female & $2248(38.0 \%)$ & $2873(36.3 \%)$ & \\
\hline Race & & & $<0.0001$ \\
\hline White & $2265(38.9 \%)$ & $3721(47.0 \%)$ & \\
\hline African American & $2387(40.3 \%)$ & $2487(31.4 \%)$ & \\
\hline Asian & $395(6.7 \%)$ & $561(7.1 \%)$ & \\
\hline Hispanic & $805(13.6 \%)$ & $1004(12.7 \%)$ & \\
\hline Other & $65(1.1 \%)$ & $143(1.8 \%)$ & \\
\hline Listed primary ESRD etiology & & & $<0.0001$ \\
\hline Glomerular disease & $1155(25.8 \%)$ & $1136(20.7 \%)$ & \\
\hline Diabetes & $257(5.8 \%)$ & $472(8.6 \%)$ & \\
\hline Hypertensive nephrosclerosis & $1790(40.0 \%)$ & $2178(39.7 \%)$ & \\
\hline Polycystic kidneys & $476(10.7 \%)$ & $682(12.4 \%)$ & \\
\hline Tubular \& Interstitial diseases & $160(3.6 \%)$ & $217(4.0 \%)$ & \\
\hline Renovascular \& Other vascular diseases & $218(4.9 \%)$ & $270(4.9 \%)$ & \\
\hline Congenital, rare familial \& metabolic disorders & $64(1.4 \%)$ & $51(0.9 \%)$ & \\
\hline Neoplasms & $0(0 \%)$ & $2(<0.1 \%)$ & \\
\hline Other & $350(7.8 \%)$ & $474(8.7 \%)$ & \\
\hline $\mathrm{ABO}$ & & & $<0.0001$ \\
\hline 0 & $2732(46.2 \%)$ & $3787(47.8 \%)$ & \\
\hline A & 1949 (32.9\%) & $2866(36.2 \%)$ & \\
\hline $\mathrm{B}$ & $954(16.1 \%)$ & $899(11.4 \%)$ & \\
\hline$A B$ & $282(4.8 \%)$ & $364(4.6 \%)$ & \\
\hline Primary insurance & & & 0.0002 \\
\hline Private & $1624(27.8 \%)$ & $2090(26.7 \%)$ & \\
\hline Medicare & $3815(65.3 \%)$ & $5313(67.8 \%)$ & \\
\hline Medicaid & $324(5.6 \%)$ & $322(4.1 \%)$ & \\
\hline Other & $76(1.3 \%)$ & $107(1.4 \%)$ & \\
\hline Peak PRA & & & $<0.0001$ \\
\hline $0-9 \%$ & $3956(67.1 \%)$ & $5752(72.9 \%)$ & \\
\hline $10-79 \%$ & $1452(24.6 \%)$ & $1850(23.4 \%)$ & \\
\hline $80-100 \%$ & $488(8.3 \%)$ & $289(3.7 \%)$ & \\
\hline Peak PRA (Mean \pm SD) & $16.7 \pm 28.3$ & $12.0 \pm 22.8$ & $<0.0001$ \\
\hline Transplant PRA & & & $<0.0001$ \\
\hline $0-9 \%$ & 4779 (84.4\%) & $6742(89.2 \%)$ & \\
\hline $10-79 \%$ & $716(12.6 \%)$ & $727(9.6 \%)$ & \\
\hline $80-100 \%$ & $170(3.0 \%)$ & $90(1.2 \%)$ & \\
\hline Transplant PRA (Mean \pm SD) & $7.8 \pm 20.3$ & $4.7 \pm 14.9$ & $<0.0001$ \\
\hline HLA mismatch (A/B/DR) & & & $<0.0001$ \\
\hline 0 & $544(9.2 \%)$ & $455(5.8 \%)$ & \\
\hline 1 & $48(0.8 \%)$ & $42(0.53 \%)$ & \\
\hline 2 & $233(3.9 \%)$ & $198(2.5 \%)$ & \\
\hline 3 & $768(13.0 \%)$ & $771(9.7 \%)$ & \\
\hline 4 & $1590(26.9 \%)$ & $1993(25.2 \%)$ & \\
\hline 5 & $1883(31.8 \%)$ & $2820(35.6 \%)$ & \\
\hline 6 & $850(14.4 \%)$ & $1635(20.7 \%)$ & \\
\hline HLA-A mismatch & & & $<0.0001$ \\
\hline 0 & $866(14.6 \%)$ & $916(11.6 \%)$ & \\
\hline 1 & $2128(35.98 \%)$ & $3040(38.4 \%)$ & \\
\hline 2 & $2923(49.4 \%)$ & $3959(50.0 \%)$ & \\
\hline HLA-B mismatch & & & $<0.0001$ \\
\hline 0 & $646(10.9 \%)$ & $598(7.6 \%)$ & \\
\hline 1 & $1423(24.1 \%)$ & $1833(23.2 \%)$ & \\
\hline 2 & $3848(65.0 \%)$ & $5484(69.3 \%)$ & \\
\hline HLA-DR mismatch & & & $<0.0001$ \\
\hline 0 & $1251(21.2 \%)$ & $900(11.4 \%)$ & \\
\hline 1 & $2728(46.1 \%)$ & $3151(39.8 \%)$ & \\
\hline 2 & $1937(32.7 \%)$ & $3864(48.8 \%)$ & \\
\hline Cold ischemia time (Mean \pm SD in h) & $18.8 \pm 9.4$ & $18.9 \pm 9.8$ & 0.48 \\
\hline
\end{tabular}

$\chi^{2}$ testing utilized, except for age, mean PRA, and cold ischemia-which utilized t-testing.

American Journal of Transplantation 2012; 12: 2106-2114 
Table 2: Recovered and transplanted kidneys by donor type and KDRI category

\begin{tabular}{|c|c|c|c|c|c|c|}
\hline \multirow[b]{2}{*}{ KDRI category } & \multicolumn{3}{|c|}{ Recovered kidneys } & \multicolumn{3}{|c|}{ Transplanted kidneys } \\
\hline & SCD & ECD & $p(\chi 2)$ & SCD & ECD & $p(\chi 2)$ \\
\hline Overall (1.4-2.1) & 8267 (84.0\%) & 11979 (89.7\%) & $<0.0001$ & $5917(71.6 \%)$ & 7916 (66.1\%) & $<0.0001$ \\
\hline $1.4-1.6$ & $5058(87.4 \%)$ & $2556(94.3 \%)$ & $<0.0001$ & 3956 (78.2\%) & $1969(77.0 \%)$ & 0.24 \\
\hline $1.6-1.8$ & $2222(81.0 \%)$ & 3963 (91.4\%) & $<0.0001$ & $1408(63.4 \%)$ & $2835(71.5 \%)$ & $<0.0001$ \\
\hline $1.8-2.1$ & $987(75.1 \%)$ & $5460(86.5 \%)$ & $<0.0001$ & 553 (56.0\%) & $3112(57.0 \%)$ & 0.57 \\
\hline
\end{tabular}

Percentage $=$ recovered or transplanted kidneys / total kidneys of that donor type.

at increased risk of discard. In fact, in the KDRI 1.6-1.8 interval, the OR of discard for ECD kidneys was 0.74 ( $p<$ 0.0001) compared to SCD kidneys.

Biopsied SCD kidneys were more frequently discarded overall $(31.8 \%$, vs. $21.1 \%$ of unbiopsied SCD kidneys, $\mathrm{p}<0.0001$, Table 4), and in the lower two KDRI categories $(15.5 \%$ and $30.7 \%$, vs. $25.4 \%$ and $38.7 \%$ for unbiopsied SCD kidneys in the lower and middle categories, $p<0.0001$ and $p=0.0005$, respectively). For the lower two KDRI categories, ECD discard rates were not significantly affected by biopsy status. In the highest KDRI category, there was not a significant difference for SCD kidneys (45.7\% vs. $43.5 \%$ for unbiopsied SCD kidneys, $p=0.59$ ), whereas the rate of discard for biopsied ECD kidneys was significantly lower than that of unbiopsied ECD kidneys (47.6\% vs. $42.4 \%, p=0.02$ ).

In the adjusted logistic model for discard (Table 5), there were significantly higher odds of both SCD and ECD dis- card when a biopsy was performed (OR 1.87, $p<0.0001$, and 1.21, $p=0.002$, respectively), but this effect was much more strongly modulated by KDRI among SCD kidneys than among ECD kidneys, such that discard practice patterns for biopsied SCD kidneys begin to mirror those for ECD kidneys in the higher KDRI categories. In contrast, the odds of ECD discard when a biopsy was performed were not affected by KDRI. Whether or not actual biopsy results (percent glomerulosclerosis) were included in the adjusted logistic modeling, rather than merely biopsy status (done/not done), the modeling was nearly identical (data not shown).

Unadjusted discard rates were lower for pumped kidneys compared with those not pumped for all KRDI categories of ECD and SCD kidneys (Table 6). The differences in discard rates between pumped and unpumped kidneys increased with increasing KDRI for both ECD and SCD kidneys, with almost half of unpumped kidneys discarded for both SCD and ECD kidneys at the highest KDRI category $(48.1 \%$ and

Table 3: Adjusted odds ratio for discard, stratified by KDRI category

\begin{tabular}{lcccccccc}
\hline Covariates & All OR & $p$ & KDRI 1.4-1.6 & $p$ & KDRI 1.6-1.8 & $p$ & KDRI 1.8-2.1 & $p$ \\
\hline ECD & 1.29 & $<0.0001$ & 1.01 & 0.89 & 0.74 & $<0.0001$ & 0.97 & 0.72 \\
Biopsy not done & 1.18 & 0.0006 & 0.95 & 0.52 & 1.23 & 0.01 & 1.89 & $<0.0001$ \\
Biopsy missing & 1.61 & 0.59 & 0.83 & 0.87 & 4.75 & 0.007 & 7.39 & $<0.0001$ \\
Glomerulosclerosis (6-10\%) & 1.44 & $<0.0001$ & 1.57 & $<0.0001$ & 1.21 & 0.05 & 1.42 & $<0.0001$ \\
Glomerulosclerosis (11-15\%) & 2.21 & $<0.0001$ & 1.94 & $<0.0001$ & 2.26 & $<0.0001$ & 2.23 & $<0.0001$ \\
Glomerulosclerosis (16-19\%) & 3.44 & $<0.0001$ & 3.82 & $<0.0001$ & 3.11 & $<0.0001$ & 3.21 & $<0.0001$ \\
Glomerulosclerosis (20\%+) & 7.67 & $<0.0001$ & 7.23 & $<0.0001$ & 7.51 & $<0.0001$ & 6.90 & $<0.0001$ \\
Glomerulosclerosis missing & 2.60 & 0.5 & 3.10 & 0.43 & 1.00 & - & 1.00 & - \\
Male sex & 1.30 & $<0.0001$ & 1.38 & $<0.0001$ & 1.32 & $<0.0001$ & 1.37 & $<0.0001$ \\
Organ not pumped & 1.66 & $<0.0001$ & 1.45 & $<0.0001$ & 1.84 & $<0.0001$ & 1.73 & $<0.0001$ \\
Organ pumped missing & 2.80 & 0.24 & 1.72 & 0.63 & 1.00 & - & 1.00 & - \\
Pump resistance measure missing & 1.11 & 0.15 & 1.11 & 0.4 & 1.24 & 0.1 & 1.04 & 0.74 \\
Donor blood type A & 1.13 & 0.001 & 1.26 & 0.0003 & 1.07 & 0.31 & 1.07 & 0.28 \\
Donor blood type B & 0.84 & 0.001 & 0.79 & 0.02 & 0.83 & 0.06 & 0.82 & 0.03 \\
Donor blood type AB & 1.54 & $<0.0001$ & 2.07 & $<0.0001$ & 1.38 & 0.05 & 1.28 & 0.09 \\
Donor has tattoo & 1.11 & 0.04 & 1.03 & 0.77 & 1.54 & $<0.0001$ & 0.88 & 0.19 \\
Donor alcohol use & 0.90 & 0.01 & 0.84 & 0.02 & 0.91 & 0.21 & 1.02 & 0.78 \\
Donor cigarette use missing & 0.86 & $<0.0001$ & 1.01 & 0.91 & 0.77 & $<0.0001$ & 0.79 & $<0.0001$ \\
Donor cocaine use & 1.28 & $<0.0001$ & 1.64 & $<0.0001$ & 1.08 & 0.48 & 0.86 & 0.19 \\
Donor use of other drugs & 1.14 & 0.006 & 1.15 & 0.06 & 1.10 & 0.27 & 1.29 & 0.004 \\
Donor CMV positive & 1.03 & 0.39 & 0.93 & 0.26 & 1.02 & 0.72 & 0.97 & 0.63 \\
Donor HBcAb positive & 1.56 & $<0.0001$ & 1.68 & $<0.0001$ & 1.31 & 0.007 & 1.45 & $<0.0001$ \\
Donor HTLV positive & 6.03 & $<0.0001$ & 7.92 & 0.006 & 4.79 & 0.04 & 5.15 & 0.05 \\
Donor HBsAg & 4.23 & $<0.0001$ & 6.64 & 0.001 & $4.2 \times 10^{9}$ & 1 & 0.85 & 0.79 \\
\hline
\end{tabular}


Table 4: Number and percentage of kidneys that were discarded, by KDRI category, donor type and biopsy status

\begin{tabular}{lrrrr}
\hline & \multicolumn{4}{c}{ Biopsy Status } \\
\cline { 2 - 5 } KDRI category & $\mathrm{SCD}$ & $\mathrm{p}$ & $\mathrm{ECD}$ & $\mathrm{p}$ \\
\hline Overall (1.4-2.1) & & & & \\
$\quad$ Not biopsied & $551(21.1 \%)$ & $<0.0001$ & $534(33.0 \%)$ & 0.43 \\
$\begin{array}{l}\text { Biopsied } \\
\text { 1.4-1.6 }\end{array}$ & $1799(31.8 \%)$ & & $3529(34.1 \%)$ & \\
$\quad$ Not biopsied & $283(15.5 \%)$ & $<0.0001$ & $92(20.7 \%)$ & 0.22 \\
$\begin{array}{l}\text { Biopsied } \\
1.6-1.8\end{array}$ & $819(25.4 \%)$ & & $495(23.4 \%)$ & \\
$\quad$ Not biopsied & $178(30.7 \%)$ & 0.0005 & $156(27.3 \%)$ & 0.51 \\
$\quad$ Biopsied & $636(38.7 \%)$ & & $972(28.7 \%)$ & \\
$\begin{array}{l}1.8-2.1 \\
\quad \text { Not biopsied }\end{array}$ & $90(45.7 \%)$ & 0.59 & $286(47.6 \%)$ & 0.02 \\
Biopsied & $344(43.5 \%)$ & & $2062(42.4 \%)$ & \\
\hline
\end{tabular}

Significance values are biopsied versus not biopsied in the same donor type and KDRI category.

$48.4 \%$, compared to $30.4 \%$ and $33.7 \%$ for pumped SCD and ECD kidneys, respectively). There were significantly lower adjusted odds of both SCD and ECD discard if kidneys were pumped (OR 0.68, $p<0.0001$, and $0.53, p<$ 0.0001 , respectively) and, similar to the biopsy analysis, the effect was much more strongly modulated by KDRI among SCD kidneys than among ECD kidneys (Table 5) such that SCD kidney discard patterns mirrored that of ECD kidneys at higher, but not lower, KDRI values.

Finally, we sought to determine if outcomes were affected to a greater degree by KDRI or by the SCD/ECD designation. Among transplanted kidneys, adjusted graft survival was worse for $E C D$ over the entire overlap range $(H R=$ 1.13 vs. $S C D, p<0.01)$, but was not significantly different within each individual KDRI category (Figure 2), suggesting that $E C D$ and $S C D$ kidneys with similar $K D R I$ values have similar outcomes. Similarly, adjusted patient survival was worse for ECD kidneys over the entire overlap range ( $H R=$ $1.25, p<0.0001)$, but was not significantly different within each individual KDRI category (Figure 3), suggesting no survival difference between ECD and SCD kidneys within a given KDRI category.
Table 6. Number and percentage of kidneys that were discarded, by KDRI category, donor type and pump status

\begin{tabular}{|c|c|c|c|c|}
\hline \multirow[b]{2}{*}{ KDRI category } & \multicolumn{4}{|c|}{ Pump status } \\
\hline & SCD & $\mathrm{p}$ & ECD & $\mathrm{p}$ \\
\hline \multicolumn{5}{|l|}{ Overall (1.4-2.1) } \\
\hline Not pumped & \multicolumn{3}{|c|}{$1871(29.8 \%)<0.00012961(38.4 \%)$} & \multirow[t]{2}{*}{$<0.0001$} \\
\hline Pumped & $479(24.1 \%)$ & & $1102(25.8 \%)$ & \\
\hline \multicolumn{5}{|l|}{$1.4-1.6$} \\
\hline Not pumped & $848(22.3 \%)$ & \multirow[t]{2}{*}{0.17} & $438(26.4 \%)$ & \multirow[t]{2}{*}{$<0.0001$} \\
\hline Pumped & $254(20.4 \%)$ & & $149(16.7 \%)$ & \\
\hline \multicolumn{5}{|l|}{$1.6-1.8$} \\
\hline Not pumped & $659(38.5 \%)$ & \multirow[t]{2}{*}{0.0009} & $848(32.9 \%)$ & \multirow[t]{2}{*}{$<0.0001$} \\
\hline Pumped & $155(30.4 \%)$ & & $280(20.2 \%)$ & \\
\hline \multicolumn{5}{|l|}{$1.8-2.1$} \\
\hline Not pumped & \multicolumn{3}{|c|}{$364(48.1 \%)<0.00011675(48.4 \%)$} & \multirow[t]{2}{*}{$<0.0001$} \\
\hline Pumped & $70(30.4 \%)$ & & $673(33.7 \%)$ & \\
\hline
\end{tabular}

Significance values are pumped versus not pumped in the same donor type and KDRI category.

\section{Discussion}

Deceased kidney donors have fallen to 2006 levels, and the number of kidneys not utilized from recovered donors remains near the recent historic peak in 2008 (7), with the lowest transplant rates among ECD. Since the ECD/SCD designation is binary and does not account for the spectrum of donor quality within each category, such data raise the concern that the ECD label itself, rather than the actual quality of the kidney, may adversely affect willingness to use these kidneys (8). In fact, Hirth et al. demonstrated that, although OPOs were recovering kidneys from donors older than 59 years more frequently than before the ECD era, transplant centers were using them with greater selectivity (5).

With this in mind, the aim of the study was to examine the relationship of the ECD label (compared to SCD) with utilization and outcomes at a given level of donor risk. We found that the ECD designation does not seem to adversely affect utilization or outcomes beyond those predicted by the KDRI. Although ECD kidneys have higher rates of discard and graft failure risk over the entire study

Table 5: Adjusted odds ratios for discard if biopsied or pumped, stratified by donor type and KDRI category

\begin{tabular}{|c|c|c|c|c|c|c|c|c|}
\hline \multirow[b]{2}{*}{ KDRI category } & \multicolumn{4}{|c|}{ Biopsied (reference $=$ unbiopsied) } & \multicolumn{4}{|c|}{ Pumped (reference $=$ nonpumped) } \\
\hline & $\mathrm{SCD}^{1}$ & $p$ & $\mathrm{ECD}^{1,2}$ & $p$ & $\mathrm{SCD}^{3}$ & $p$ & $\mathrm{ECD}^{3,4}$ & $p$ \\
\hline Overall (1.4-2.1) & 1.87 & $<0.0001$ & 1.21 & 0.002 & 0.68 & $<0.0001$ & 0.53 & $<0.0001$ \\
\hline $1.4-1.6$ & 1.93 & $<0.0001$ & 1.20 & 0.18 & 0.76 & 0.005 & 0.54 & $<0.0001$ \\
\hline $1.6-1.8$ & 1.58 & $<0.0001$ & 1.25 & 0.04 & 0.64 & 0.0007 & 0.48 & $<0.0001$ \\
\hline $1.8-2.1$ & 1.06 & 0.73 & 1.00 & 0.98 & 0.54 & 0.001 & 0.52 & $<0.0001$ \\
\hline
\end{tabular}

${ }^{1}$ A stratified test for trend showed significant KDRI $*$ biopsy (est. $=-0.28 ; p=0.001$ ) interaction among SCD kidneys. Nonsignificant results were shown among ECD kidneys.

${ }^{2}$ Analysis for three-way interaction showed nonsignificant $\mathrm{KDRI} * \mathrm{ECD} *$ biopsy interaction (est. $=0.14 ; \mathrm{p}=0.224$ )

${ }^{3} \mathrm{~A}$ stratified test for trend showed significant KDRI $*$ pump (est. $=-0.17 ; p=0.046$ ) interaction among SCD kidneys. Nonsignificant results were shown among ECD kidneys.

${ }^{4}$ Analysis for three-way interaction showed marginally significant KDRI $* E C D *$ pump interaction (est. $=0.20 ; p=0.060$ ). 

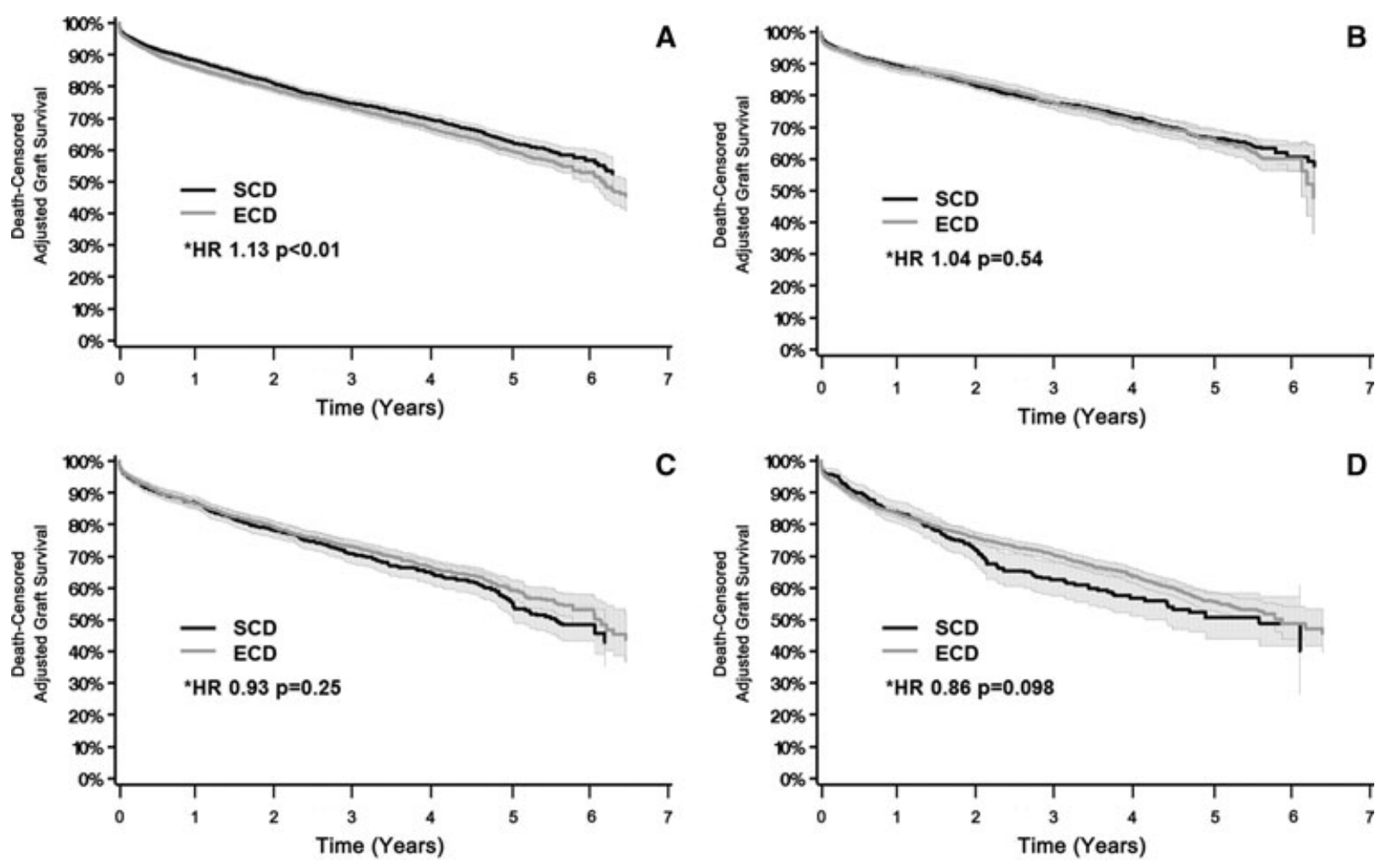

Figure 2: Death-censored adjusted graft survival by donor type and KDRI category, with $\mathbf{9 5 \%}$ confidence intervals. (A) Overall KDRI 1.4-2.1. (B) KDRI 1.4-1.6. (C) KDRI 1.6-1.8. (D) KDRI 1.8-2.1.

population, within a given KDRI interval, the ECD designation does not confer additional risk of discard or graft failure compared with SCD kidneys within the same interval. This apparent paradox may be explained by decreasing utilization and graft survival rates attendant with increases in KDRI (regardless of whether kidneys are ECD or SCD), and the differently skewed distributions of KDRI values for ECD and SCD kidneys in the overlap range (a greater proportion of ECD kidneys in the higher KDRI interval, and of SCD kidneys in the lower interval, Figure 1). Stratifying the study population (the overlap region of SCD and ECD kidneys) by intervals of KDRI was critical to this analysis, as it eliminated the effect of unequal distributions of KDRI among SCD and ECD kidneys. Thus, the average of the averages of these groups is not the same as the average of the combined population.

However, the ECD label does appear to affect perceptions of donor quality in that the effects of biopsy and pumping on discard are influenced by KDRI to a greater degree among SCD, compared to ECD kidneys. This results in similar discard practices attributable to biopsy (higher discard odds) and pumping (lower discard odds) for higher risk SCD kidneys and lower risk ECD kidneys.

ECD kidneys have been shown to have a survival benefit compared to dialysis, and even compared with waiting for an SCD kidney for specific classes of recipients (patients older than 40 years in OPOs with $>1350$ days of waiting list time; diabetic patients older than 40 years) (4). Despite this, ECD designation practices vary widely, with only about half of those predicted to benefit from ECD actually so designated $(3,9)$. Although patient and graft survival for ECD kidneys are lower than for SCD kidneys (10), this can be offset by earlier ECD transplant for selected subgroups $(3,4)$. Although our data demonstrated lower adjusted patient and graft survival overall, this finding was (as was the case with utilization) primarily because ECD kidneys tended to be at the higher end of the KDRI spectrum, even in our overlap region, whereas SCDs tended to be in the lower end. In each KDRI category, survival was not significantly different between ECD and SCDs, supporting the conclusion that the ECD designation does not predict outcomes beyond that predicted by KDRI.

Over a third of discarded kidneys are discarded due to biopsy findings (7). Similar to previous analyses (11), we found that, while actual biopsy results (e.g. percentage glomerulosclerosis) strongly impacted discard rates, having any biopsy performed resulted in increased discard rates throughout the spectrum of our KDRI range for both SCD and ECD kidneys. However, KDRI influenced this biopsy effect for SCD kidneys, but not ECD kidneys, and as the KDRI value rises for SCD kidneys, the effect of biopsy 


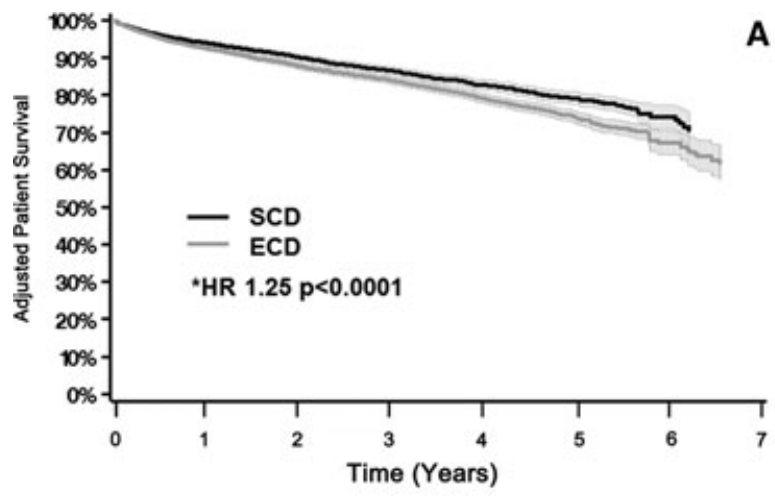

A
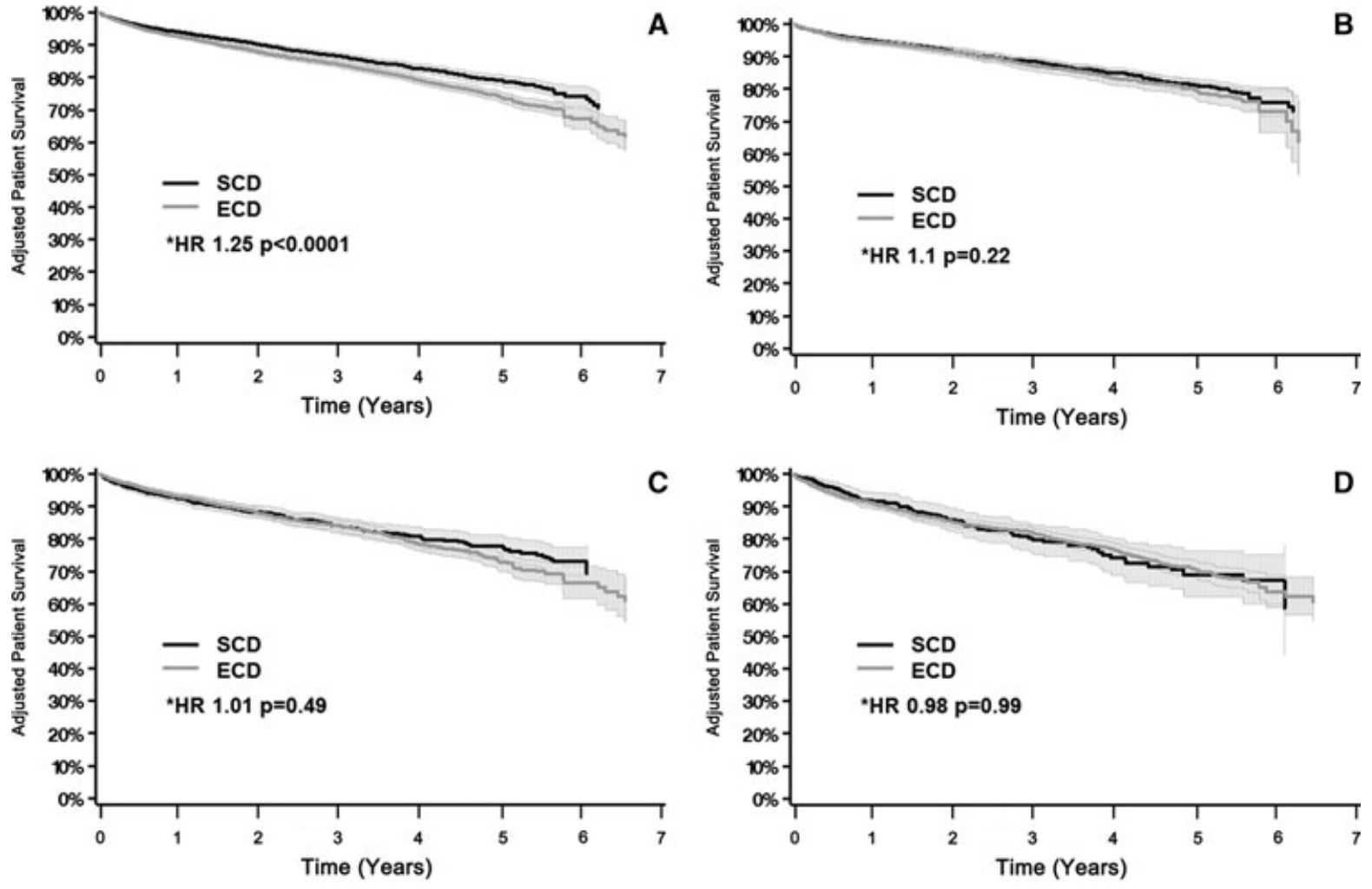

Figure 3: Adjusted patient survival by donor type and KDRI category, with $\mathbf{9 5 \%}$ confidence intervals. (A) Overall KDRI 1.4-2.1. (B) KDRI 1.4-1.6. (C) KDRI 1.6-1.8. (D) KDRI 1.8-2.1.

on discard seems to approach that of ECD kidneys. SCD biopsy is usually not protocolized, but more frequently performed for indication. In contrast, many OPOs biopsy all ECD kidneys as a policy, which may account for the weak relationship observed between biopsy and discard for ECD kidneys. In our current study, we did not analyze outcomes based on biopsy findings, and the impact of glomerulosclerosis and other biopsy parameters on transplant outcomes remains controversial (11).

In contrast, there is increasingly convincing data from both observational studies and prospective trials that pulsatile perfusion has a positive effect on outcomes. Pumped kidneys are discarded less frequently, are significantly less likely to have delayed graft function (DGF), and were found to have improved 1-year graft survival in prospective trials (12). Favorable pump parameters strongly correlate with lower discard rates. The overall impact of pumping on utilization is difficult to discern because pumping practices vary widely. Nevertheless, pumping was associated with a lower discard rate for the kidneys in the KDRI overlap region for all subgroups. For ECD kidneys, there was a constant relationship between pumping and discard rates across the KDRI spectrum, whereas for SCD kidneys an increasing inverse association between pumping and discard with increasing KDRI was observed. Similar to biopsy, many OPOs pump ECD kidneys as a matter of proto- col, whereas SCD kidneys are more frequently pumped by request or indication, which may account for the incremental effect on kidney discard that was seen with increasing $\mathrm{KDRI}$ for SCD kidneys but not observed for ECD kidneys.

This study has a number of limitations. As it uses registry data, it is dependent upon its accuracy and completeness, and analyzes outcomes in a retrospective manner. As the transplant outcomes data are based on utilized kidneys, the study's conclusions about the quality of ECD kidneys at a given KDRI are best applied to kidneys accepted under current utilization patterns. It is not known if discarded kidneys from SCD versus ECD donors would have similar outcomes if utilized. Although the overall cohort size is large, the multiple covariates and indicator variables in a stratified analysis leave open the possibility of Type 2 statistical error, especially for the biopsy and pumping analyses.

The findings also support the elements of the proposed kidney allocation system that designate organ quality based on a kidney donor profile index (DPI), which ranks donor kidneys based on KDRI, rather than ECD/SCD, since the ECD designation does not appear to add information regarding donor risk beyond that conferred by the KDRI. These results suggest that, in a KDRI-based allocation system, 
the elimination of the ECD designation would not have a significant impact, provided there was a mechanism for older recipients or those with poor expected waiting list time or posttransplant survival to be able to receive higher KDRI organs.

However, ECD does appear to override other estimates of donor quality (such as the other elements of KDRI), in the utilization of biopsied and pumped kidneys; in other words, behavior for ECD kidneys is similar regardless of KDRI, whereas KDRI affects behavior for SCD such that only in the highest KDRI category studied do SCD and ECD kidneys appear to be handled similarly. Elimination of the ECD designation may potentially improve utilization for lower risk kidneys currently labeled ECD.

In conclusion, the ECD designation does not seem to adversely affect utilization or outcomes beyond that predicted by the KDRI. Within a given KDRI category, the ECD designation does not confer an increased graft failure risk compared with SCD kidneys. The effects of biopsy and pumping on discard are modulated by KDRI to a greater degree among SCD, compared to ECD, kidneys. This results in similar discard practices attributable to biopsy (higher discard odds) and pumping (lower discard odds) among higher risk SCD kidneys and lower risk ECD kidneys.

\section{Acknowledgments}

The SRTR is funded by contract number 234-2005-37009C from the HRSA, US Department of Health and Human Services. The views expressed herein are those of the authors and not necessarily those of the US Government. This is a US Government-sponsored work. There are no restrictions on its use.

\section{Disclosure}

The authors of this manuscript have no conflicts of interest to disclose as described by the American Journal of Transplantation.

\section{References}

1. Port FK, Bragg-Gresham JL, Metzger RA, et al. Donor characteristics associated with reduced graft survival: An approach to expanding the pool of kidney donors. Transplantation 2002; 74: 1281-1286.

2. Tuttle-Newhall JE, Krishnan SM, Levy MF, McBride V, Orlowski JP, Sung RS. Organ donation and utilization in the United States: 1998-2007. Am J Transplant 2009; 9: 879-893.

3. Grams ME, Womer KL, Ugarte RM, Desai NM, Montgomery RA, Segev DL. Listing for expanded criteria donor kidneys in older adults and those with predicted benefit. Am J Transplant 2010; 10: 1-8.

4. Merion RM, Ashby VB, Wolfe RA, et al. Deceased-donor characteristics and the survival benefit of kidney transplantation. JAMA 2005; 294: 2726-2733.

5. Hirth RA, Pan Q, Schaubel DE, Merion RM. Efficient utilization of the expanded criteria donor (ECD) decreased donor kidney pool: An analysis of the effect of labeling. Am J Transplant 2010; 10: 304-209.

6. Rao PS, Schaubel DE, Guidinger MK, et al. A comprehensive risk quantification score for deceased donor kidneys: The Kidney Donor Risk Index. Transplantation 2009; 88: 231236.

7. Klein AS, Messersmith EE, Ratner LE, Kochik R, Baliga PK, Ojo AO. Organ donation and utilization in the United States, 19992008. Am J Transplant 2010; 10: 973-986.

8. Delmonico FL, O'Connor K, Krishnan SM, et al. ECD kidney transplant outcomes do not justify the national rate of ECD kidney discard or the loss of functioning ECD kidneys by patient deaths. Am J Transplant 2010; 10 (Suppl): s71.

9. Schold JD, Howard RJ, Scicchitano MJ, Meier-Kriesche HU. The expanded criteria donor policy: An evaluation of program objectives and indirect ramifications. Am J Transplant 2006; 6: 16891695.

10. Wolfe RA, Roys EC, Merion RM. Trends in organ donation and transplantation in the United States, 1999-2008. Am J Transplant 2010; 10: 961-972.

11. Sung RS, Christensen LL, Leichtman AB, et al. Determinants of discard of expanded criteria donor kidneys: Impact of biopsy and machine perfusion. Am J Transplant 2008; 8: 783792.

12. Moers C, Smits JM, Maathuis MJ, et al. Machine perfusion or cold storage in deceased-donor kidney transplantation. $\mathrm{N}$ Engl J Med 2009; 360: 7-19. 